\title{
Personal Health Care System Using IOT
}

\author{
https://doi.org/10.3991/ijoe.v15i07.10265 \\ Hatem Elaydi \\ Islamic University of Gaza, Gaza, Palestine \\ helaydi@iugaza.edu.ps
}

\begin{abstract}
Designing a device to manage the medical doses for elderly people is essential in terms of the patient safety and well-being. The device is created in order to regulate medication schedules and deliver doses of medication according to a prescribed time and dose by alerting patients of the scheduled dose through communication means such as mobile phones. The device utilizes mobile technology and communication such as Android and Wi-Fi. This device consists of six basic elements: Arduino Uno board, ESP8266 Wi-Fi module that connects the phone with the device, RTC module (Real Time Clock), GSM module for alarming when the time of medication is reached, Drivers for motors to release the prescribed doses of the medication and Smartphone for control purposes. The device was built and tested and showed promise to be used for serving the local community.
\end{abstract}

Keywords-Medical doses, Arduino, smart phones, elderly, automation

\section{Introduction}

Home automation involves the integration of technology and services within the home, with the aim of improving the quality of living using improved security, comfort, communication and management of technology. The rapid growth of information technology and the trend of aging society make medical and health services more popular [1]. But, there are still problems that need to be solved, such as choosing networking protocol, selecting sensors and wireless connections [2].

Treating older persons with dignity and respect reflects cultural values in Palestinian society. As life expectancy increases, older people want to play active role in their families and want to gain independence in their daily activities. Modern information technology tools and software enables elderly people to take charge of their lives such as to adhere to their medication schedule which becomes a challenge as their memories diminish. Elderly people develop some bad habits such as medicine sharing, taking more than the required medical doses, and sometimes forgetting about the doses.

Automatic dose dispensing machines (ADDM), devices loaded with unit-dose bags for single-time usage [3], can solve these problems. ADDMs are not new in the medical world, they are used in primary healthcare in Sweden, Denmark, Norway, and Finland as early as 2000 [4], [5], [6], [7]. ADDMs was used effectively in Nordic countries for elderly prime healthcare patients to ensure safe medication [8], [9]. 
ADDM use resulted in enhancing patient safety, decreasing medication costs, and saving nurses working time in the primary healthcare [10].

In Arabic culture, older people are respected and venerated for their wisdom, but modern technology may affect this tradition, since younger generations are more adapted to modern life. The Palestinian society is widely considered a young society with high birth rate and low percentage of the elderly. In 2017, the Palestinian elderly population, aged 60 and over, reached $4.6 \%$ of the population in Palestine $(5.1 \%$ in West Bank and 3.9\% in Gaza Strip) [11]. Over the last two decades, life expectancy in Palestine increased from 67 years in 1992 to 74 years by 2017 and is expected to continue increasing to reach 74.3 by 2020 [12].

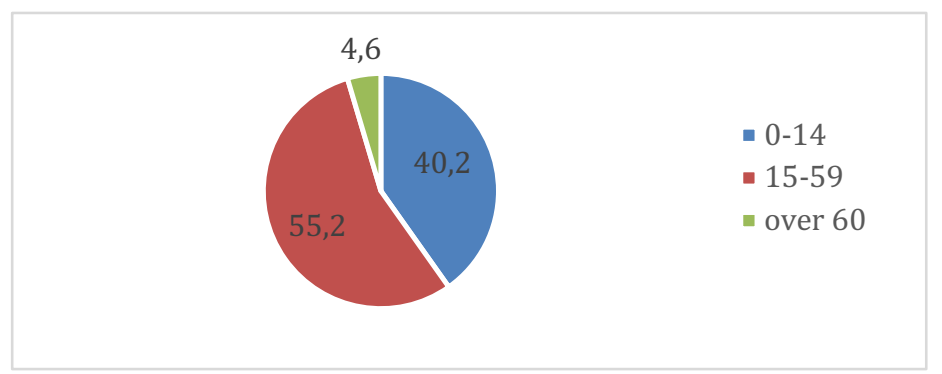

Fig. 1. The Palestinian population segmentation 2016

Palestine is experiencing lifestyle shifts such as an increase in nuclear families living alone instead of in the same home with their extended families and an increase in numbers of women participating in the labor force [12]. This led to increased number of elderly people who are living alone or left alone at homes with limited support systems.

Elderly people are usually suffer from chronic diseases that force them to lose their independence. According to 2012 WHO EMRO, Palestine had the crude birth rate of 27.1 births per 1000 persons, and the crude death of 2.7 deaths per 1000 persons [13]. The six leading causes of death are heart disease $(21.0 \%$ of all deaths), cerebrovascular disease $(11.0 \%)$, cancer $(10.3 \%)$, conditions in perinatal period $(8.6 \%)$, respiratory disorders (6.9\%) and accidents (5.4\%) [14]. The majority (97\%) of elderly in Palestine are cared for by their children [15].

Thus, there is an existing need to develop a system with module and method that provides tracking and reminder of medication dosage using sensors, mobile applications and cloud services. This research proposes building an ADDM for the purpose of improving medical adherence through proper monitoring system. By integrating a database system with the ADDM, real-time tracking is possible which helps in monitoring medical dosage, generating medicine orders and producing medical reports for doctors. This research aims to design a model that increases medical adherence amongst elderly patients, to develop and deploy low cost sensor based system for medical tracking and reminder system, to develop an efficient way to communicate between embedded system and cloud services, and to improve the existing medication reminder system by integrating tracking and cloud functionality. 
This paper is organized such as: section 2 talks about the system main components, section 3 presents project's algorithm and working principles, section 4 presents the results and shows the system as a whole, and section 5 concludes this paper.

\section{Main Components}

This section describes designing a medication release system for taking medication on time with the exact prescribed dose. The system can be controlled through mobile technology based on android software. The developed system is intended to provide alerting messages around dose times and provides the exact quantities throughout the day.

The system controls the medical doses remotely and consists of six basic components, as shown in Fig. 2, such as: smartphone, Wi-Fi module (ESP8266), RTC (DS1307) the Arduino board, Ultrasonic, and the motor's drivers. The control passes through three main stages: first, the smartphone connects to the Wi-Fi module; then, the Wi-Fi module passes the commands and the time of the smartphone to the Arduino via a serial communication port; in the third step, the Arduino reads the time of RTC module and control the device (lower the doses on-time) via motor's drivers and sends an alarm through GSM module.
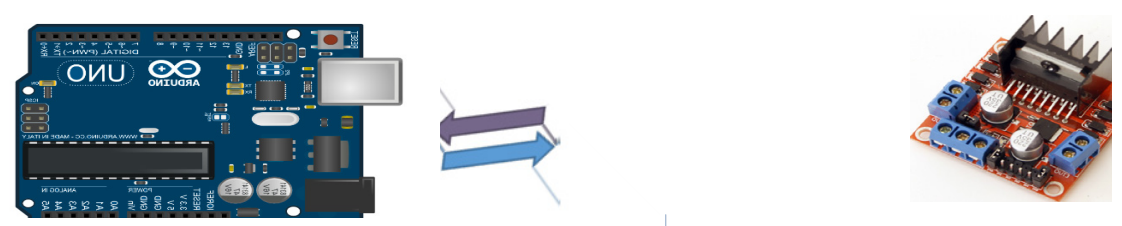

Fig. 2. The block diagram of the proposed system

Arduino Uno is a microcontroller board based on the ATmega328P as shown in figures 3 and 4 [16]. It contains everything needed to support the microcontroller; simply connect it to a computer with a USB cable or power it with an AC-to-DC adapter or battery to get started. Arduino Integrated Development Environment, IDE, is used to write programming codes in the Arduino $\mathrm{C}$ language, then convert them to an executive formula to be burned to the microcontroller in the Arduino board. ESP8266 or ESP is a Wi-Fi module suitable for supporting the Wi-Fi protocol, supporting the TCP/IP (transition control protocol/ internet protocol), and it can even be programmed as an access point as shown in Fig. 5 [17]. 


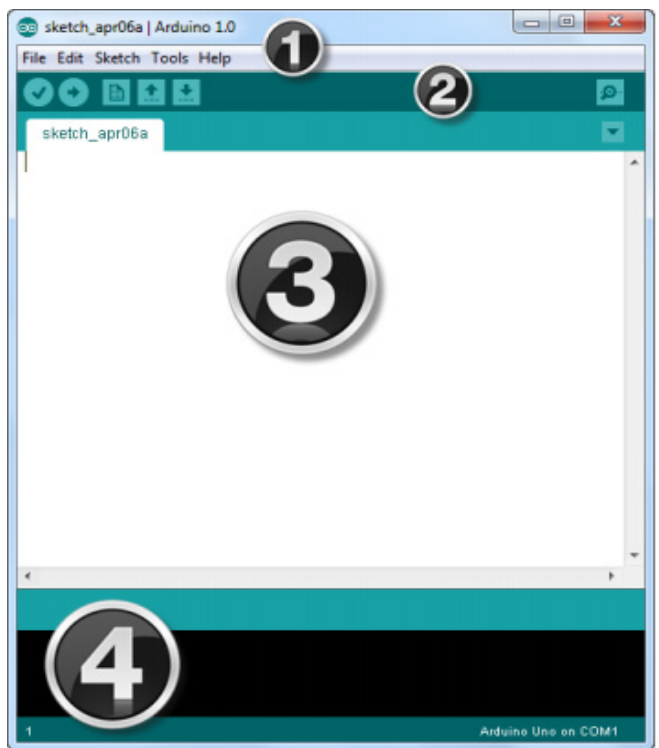

Fig. 3. Arduino IDE

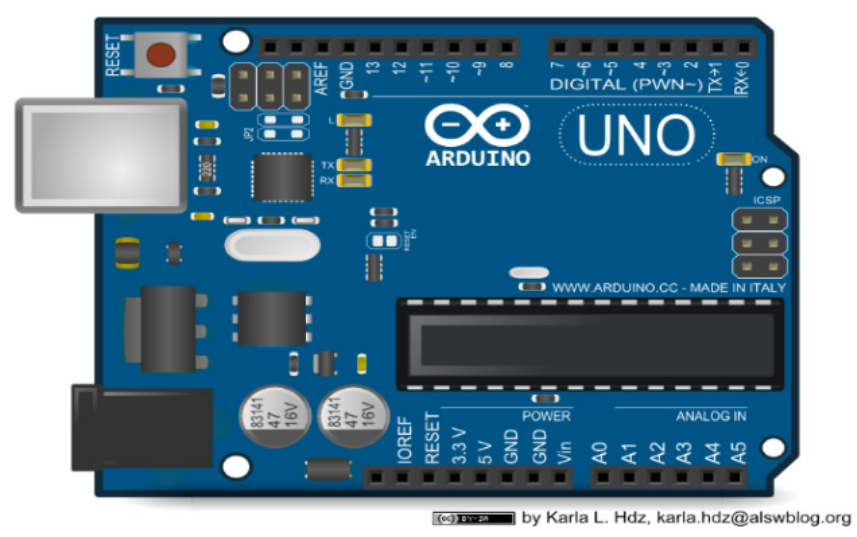

Fig. 4. The Arduino board

ESP8266 or ESP is a Wi-Fi module suitable for supporting the Wi-Fi protocol, supporting the TCP/IP (transition control protocol/ internet protocol), and it can even be programmed as an access point as shown in Fig. 5 [17]. 


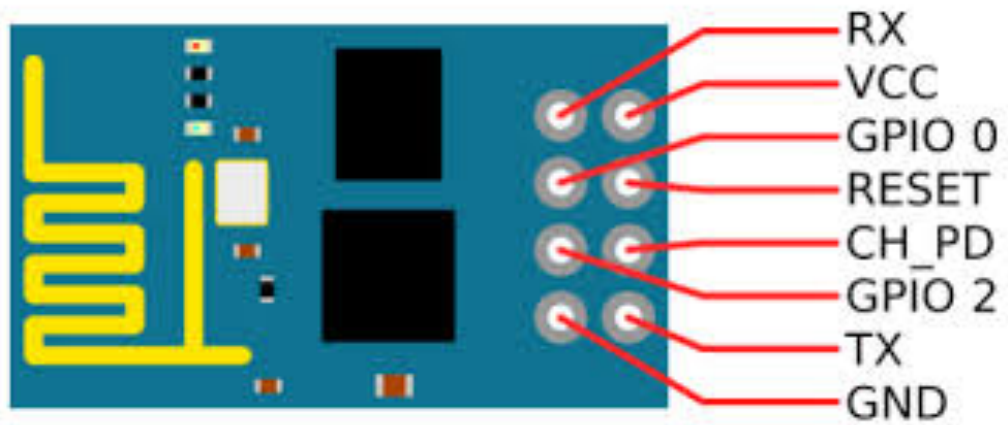

Fig. 5. The ESP8266
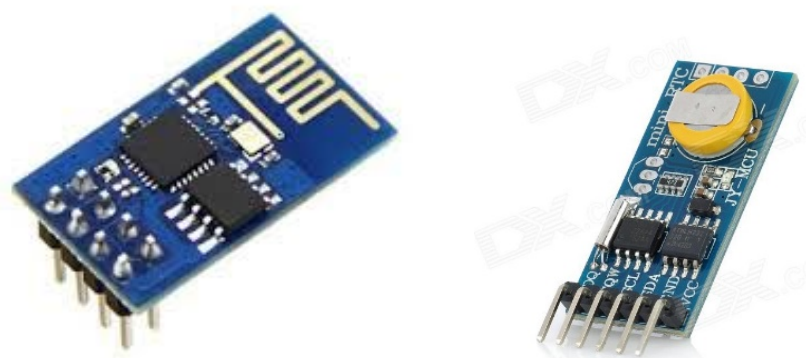

Fig. 6. DS1307

The DS1307 is serial real-time clock (RTC) as shown in Fig. 6 [18]. Address and data are transferred serially through an $\mathrm{I} 2 \mathrm{C}$, bidirectional bus. The clock/calendar provides seconds, minutes, hours, day, date, month, and year information.

The Neo_ M590 is a single-chip GPRS industrial wireless module with data only as shown in Fig. 7 [19]. It has SMS, data service and other functions. The Neo_ M590 GPRS modem provides mainly SMS services and GPRS connectivity.

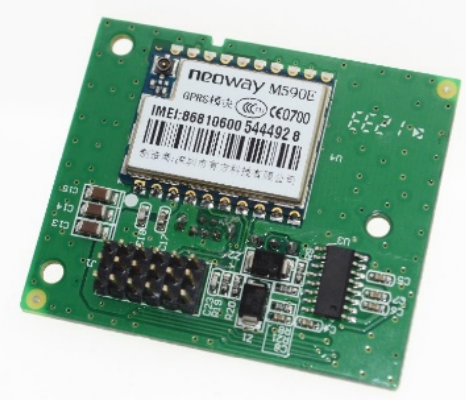

Fig. 7. GSM/GPRS module Neo_M590 
L298N Dual H-bridge Motor Controller is shown in Fig. 8 [20]. It is typically used in controlling motors speed and direction, but can be used for other projects such as driving the brightness of certain lighting projects such as high powered LED arrays. It is a circuit that can drive a current in either polarity and be controlled by Pulse Width Modulation (PWM).

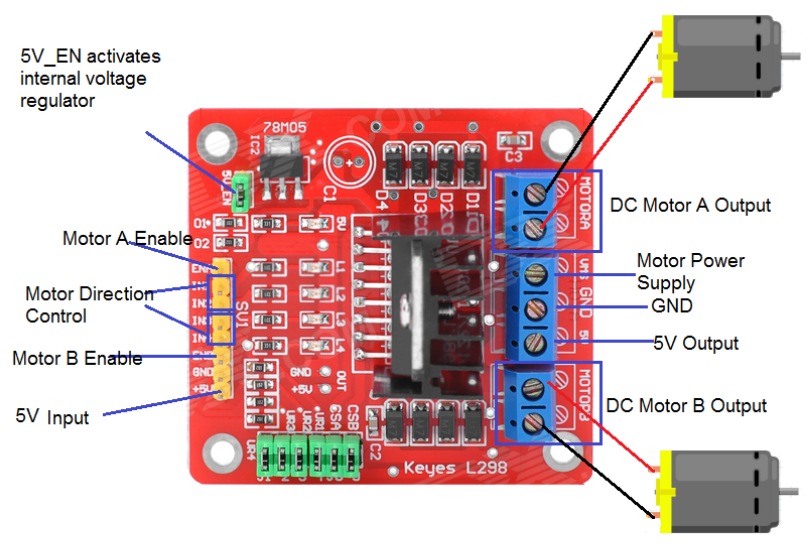

Fig. 8. Motor driver pin configuration

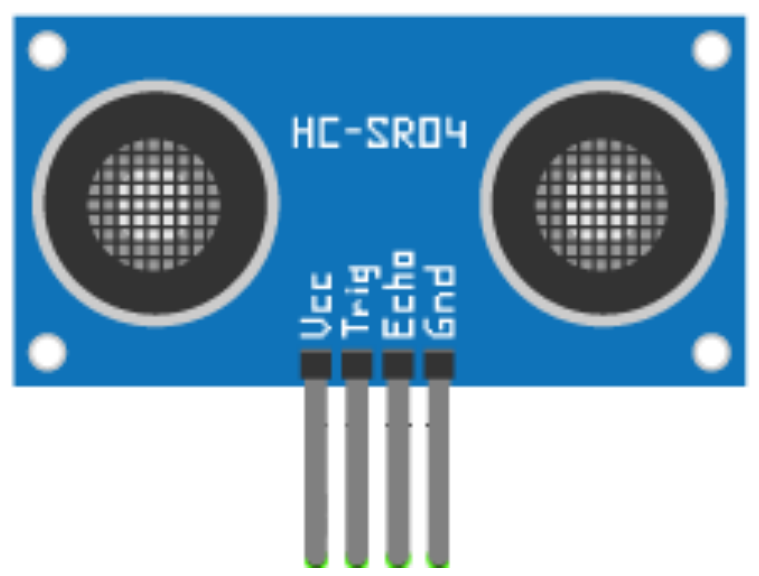

Fig. 9. Ultrasonic HC-SR04

The ultrasonic module from Arduino, as shown in Fig. 9, detects the distance of the closest object in front of the sensor (from $3 \mathrm{~cm}$ up to $400 \mathrm{~cm}$ ) [21]. Depending on the time of sending and receiving ultrasound wave.

The connection between the H-Bridge and Arduino board and Motors is listed in Table 1 and Table 2 and is shown in Fig. 10 and Fig. 11. Driver A +Arduino connections. 
Table 2. Driver A + Arduino connections

\begin{tabular}{|l|l|l|}
\hline \multicolumn{1}{|c|}{ Priver 1} & \multicolumn{1}{c|}{ Pins of driver } & \multicolumn{1}{c|}{ Motors } \\
\hline Out 1,2 & & Motor A \\
\hline Out 2,3 & & Motor B \\
\hline IN1 & 7 (Digital Pin) & - \\
\hline IN2 & 0 (GND) & - \\
\hline IN3 & 0 (GND) & - \\
\hline IN4 & 8 (Digital Pin) & - \\
\hline ENA & $5(\mathrm{PWM})$ & - \\
\hline ENB & $6(\mathrm{PWM})$ & - \\
\hline
\end{tabular}

Table 3. Driver B + Arduino connections

\begin{tabular}{|c|c|c|}
\hline \multicolumn{3}{|c|}{ Driver 2} \\
\hline Pins of driver & Pins of Arduino & Motors \\
\hline Out 1,2 & - & Motor C \\
\hline Out 2,3 & - & Motor D \\
\hline IN1 & 11 (Digital Pin) & - \\
\hline IN2 & 0 (GND) & - \\
\hline IN3 & 0 (GND) & - \\
\hline IN4 & 12 (Digital Pin) & - \\
\hline ENA & 9 (PWM) & - \\
\hline ENB & 10 (PWM) & - \\
\hline
\end{tabular}

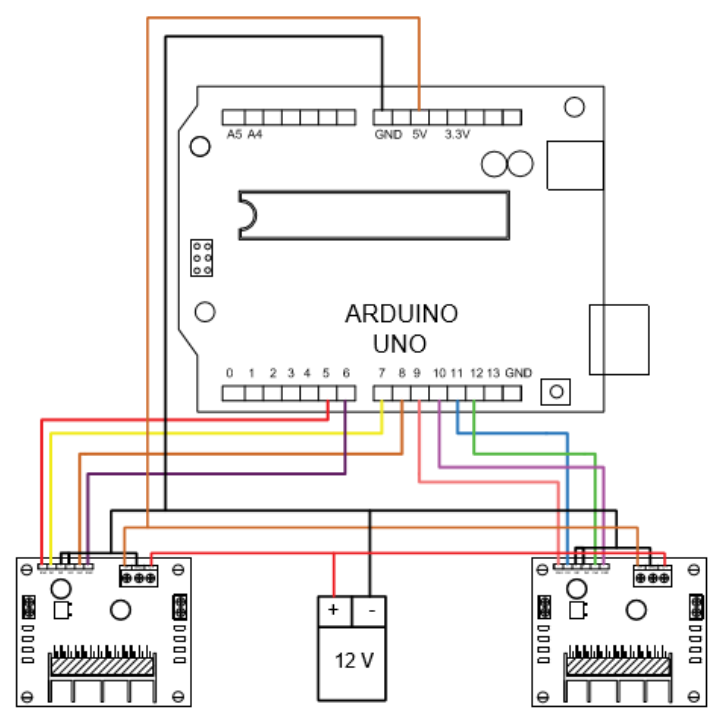

Fig. 10.Drivers Arduino connections 


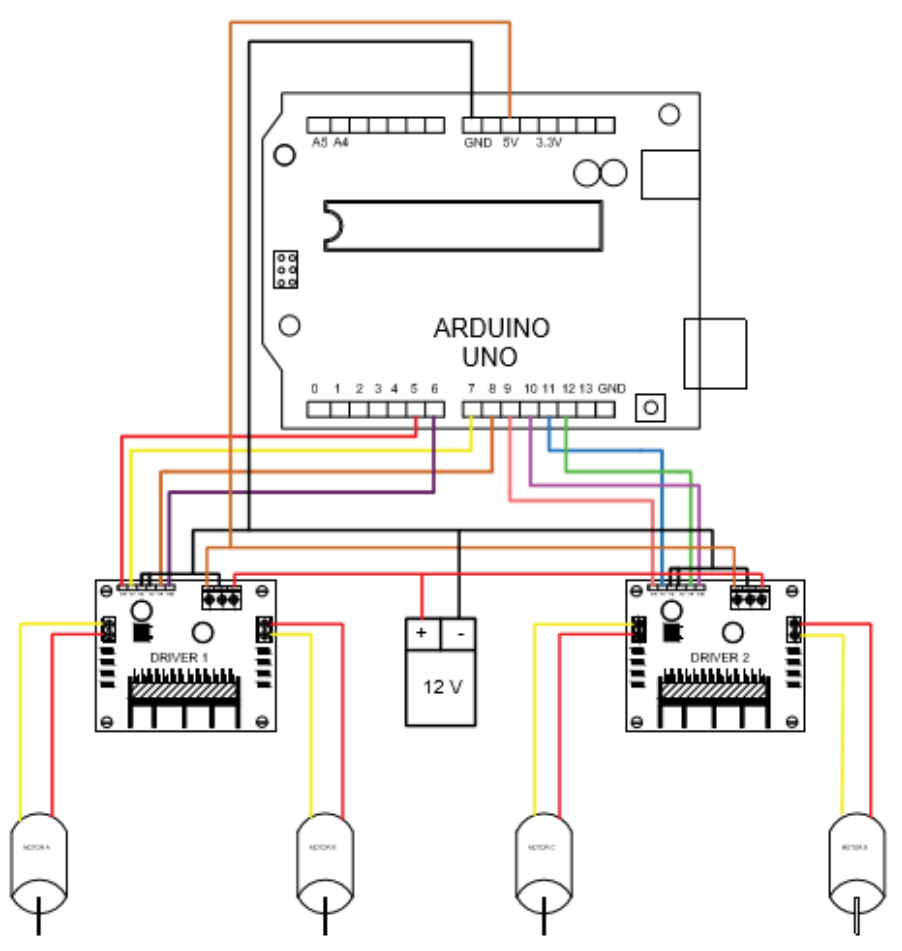

Fig. 11.Drivers +Motors connections

\subsection{Arduino board and GSM module}

The GSM is used to send alarm on scheduled dosses time of medication, moreover, this alarm also sends SMS message to the patient or nurse. The connection of GSM requires 4 pins from Arduino as shown in Table 3.

Table 4. GSM + Arduino connections

\begin{tabular}{|c|l|l|}
\hline Pin & \multicolumn{1}{|c|}{ Pins of GSM module } & \multicolumn{1}{c|}{ Pins of Arduino board } \\
\hline 1 & Vbat & 5 volt pin \\
\hline 2 & GND & GND pin \\
\hline 3 & Ring & GND pin \\
\hline 4 & GND & GND pin \\
\hline 5 & TXD & A2 (analog pin) \\
\hline 6 & RXD & A3 (analog pin) \\
\hline
\end{tabular}




\subsection{Arduino board and ultrasonic}

The ultrasonic module from Arduino detects the distance of the closest object in front of the sensor. It has 4-Pins as mentioned and is connected to the Arduino as listed in Table 4 and shown in Fig. 12.

Table 5. Ultrasonic +Arduino connections

\begin{tabular}{|c|l|l|}
\hline Pin & \multicolumn{1}{|c|}{ Pins of ultrasonic } & \multicolumn{1}{c|}{ Pins of Arduino } \\
\hline 1 & Trigpin & 4 (Digital pin) \\
\hline 2 & Echopin & 13 (Digital pin) \\
\hline 3 & Vcc & 5 volt pin \\
\hline 4 & GND & GND pin \\
\hline
\end{tabular}

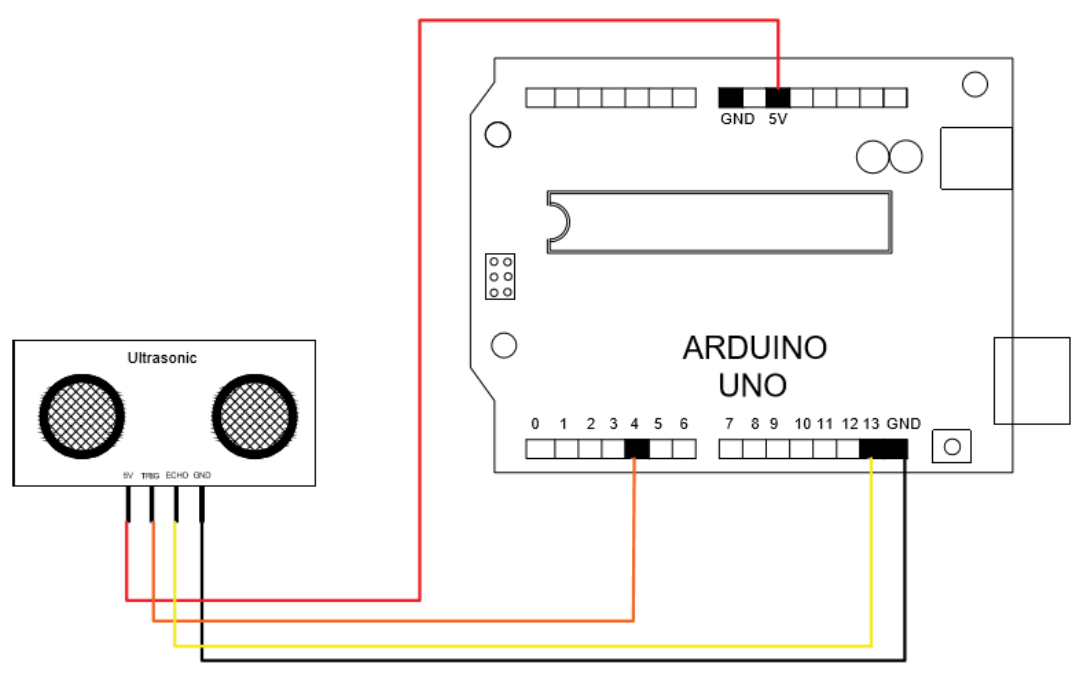

Fig. 12.Ultrasonic +Arduino connections

\section{$3 \quad$ Algorithms and Working Principles}

After explaining the electrical wiring between components of the device, and how they work. This section presents the used algorithms and how they are used in this device. In addition to explaining how to establish communication between the various components. The following connections were established:

- Arduino board and ESP8226

- Arduino board and RTC module

- Arduino board and motor driver (H-Bridge)

- Arduino board and Ultrasonic module

- Arduino board and GSM module 


\subsection{Arduino board and ESP8226}

The main commands and functions that have been used in programming are shown in Table 5.

Table 6. ESP commands

\begin{tabular}{|l|l|l|}
\hline & \multicolumn{1}{|c|}{ Command } & \multicolumn{1}{c|}{ Its work } \\
\hline 1 & SoftwareSerial espserial $(2,3)$ & Define UART serial protocol at pins 2,3 \\
\hline 2 & espserial.begin $(9600)$ & Define the baud rate of module \\
\hline 3 & & $\begin{array}{l}\text { Set the network mode to: } \\
\text { 0-Station mode } \\
\text { 1-Access point mode } \\
\text { 2-both }\end{array}$ \\
\hline 4 & AT+CWMODE & Create the name of the ESP access point \\
\hline 5 & AT+CWSAP & Set the connection mode to either single or multi \\
\hline
\end{tabular}

The functions used to connect Arduino with ESP are:

- Espread function: used to read data from ESP that is received by Arduino.

- Espsend function: used to write to ESP by Arduino to send it.

\subsection{Arduino board and RTC module}

There are two specified pins in Arduino board (SDA, SCL) that deals with I2C devices. It also has a specified library and specified commands for read and write from/to RTC module. The RTC module has specified title in Arduino programming because a huge collection modules work with $\mathrm{I} 2 \mathrm{C}$. The main commands and functions that has been used in programming are:

- Wire library: library for I2C devices

- RTC 0X86: title of RTC module

- Readtime function: read the time from RTC module

- Settime function: write and set the time to RTC module

\subsection{Arduino board and motor driver (H-Bridge)}

In this project, commands were used to send $(0,1)$ and PWM (analog output). The main commands and functions that were used in programming are:

- PinMode (pin,OUTPUT): defines the pins of Arduino boards that connected with drivers .

- DigitalWrite (pin,HIGH): defines the pins that have to be 1 (5volt)

- DigitalWrite (pin,LOW): defines the pins that have to be 0 (0volt)

- AnalogWrite (pin,0-255): defines the pins that its value have to be $0-255$ (0-5volt) analogoutput 


\subsection{Arduino board with GSM module}

The GSM module is programmed by AT-commands and Arduino board is used for sending these commands automatically when the times of medicine comes. The commands is sent from Arduino to the GSM by using UART protocol at pins (A2, A3). This command contains the message content and the text number that the message will be sent to. The main commands and functions that we have used in programming:

- AT: wake up GSM module

- $\mathrm{AT}+\mathrm{CMGF}=1$ put the $\mathrm{GSM}$ on text mode

- $\mathrm{AT}+\mathrm{CMGS}=$ give the mobile number to the GSM

\subsection{Arduino board with Ultrasonic module}

The Arduino board sends a short pulse to trigger the detection, then listens for a pulse on the same pin using the pulseIn() function, which is a specified function for this procedure. The duration of this second pulse is equal to the time taken by the ultrasound to travel to the object and back to the sensor.

\section{The Mobile Application of the Device}

Phones that support Android and IOS operating systems are utilized. An application that supports these operating systems were designed to control the device remotely, through an app. The phone app to control the device is designed using Unity program, a GUI (graphical user interface). The unity3D is used to design the control program, the GUI (graphical user interface) capabilities of the engine is used to create its multi pages, and to use the TCP/IP sockets to directly and without delay control the Arduino.

\subsection{Application description}

The application consists of 5 pages that describes the system as shown in Fig. 13 and Fig. 14.

Patient page: In this page the patient enters the main page of application without password, anyone can enter this page, without entering of password. This page is a monitoring screen for the remaining time of dosses of medicine only (can't set anything in the APP). This page is shown in Fig. 13.

Entering page: This page, as shown in Fig. 15, can be reached by pressing the button in the monitoring page (the button has doctor image). This page links users to the setting page and emergency page but this window has password which the admin and nurse only know it. 


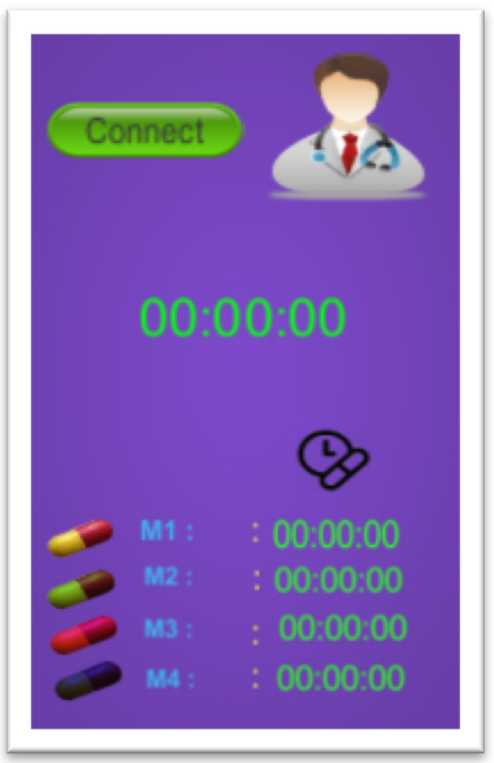

Fig. 13.Layout1- main screen

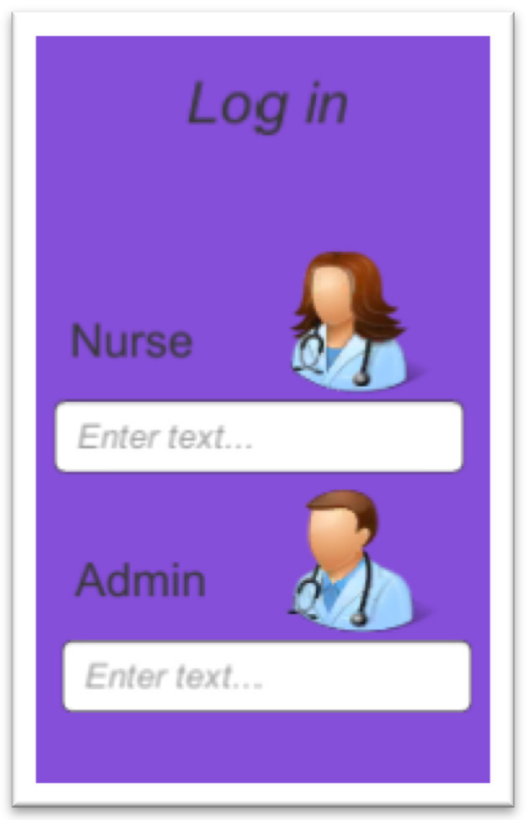

Fig. 14.Layout 2 _login 
Setting page: This page can be accessed only by the nurse and the admin, as shown in Fig. 16, by entering the password. In the input field of entering page: input field of the nurse to the nurse password and the input field of admin. In this page, the nurse or admin can set the time of doses of medicine, the name and set the number of doses per the day. In this page, there is a button, if the person presses it, it gives them accesses to the emergency page. Also, in this page the button only appears to the admin that give access to the admin's page.

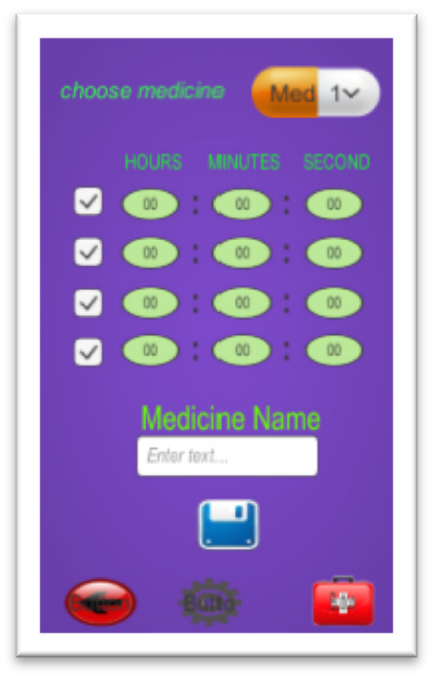

Fig. 15.Layout 3-Settings

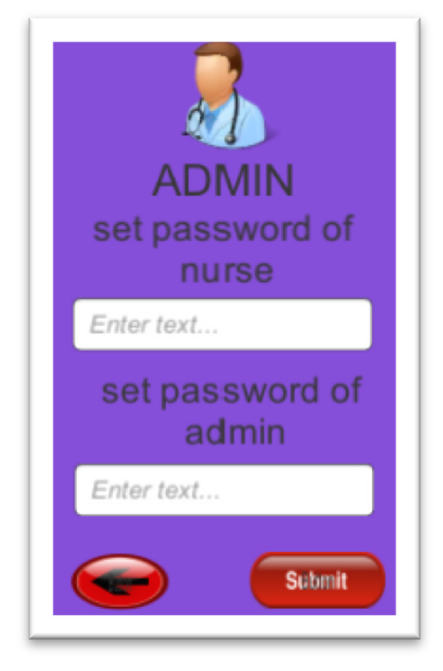

Fig. 16.Layout 4 -Password settings 


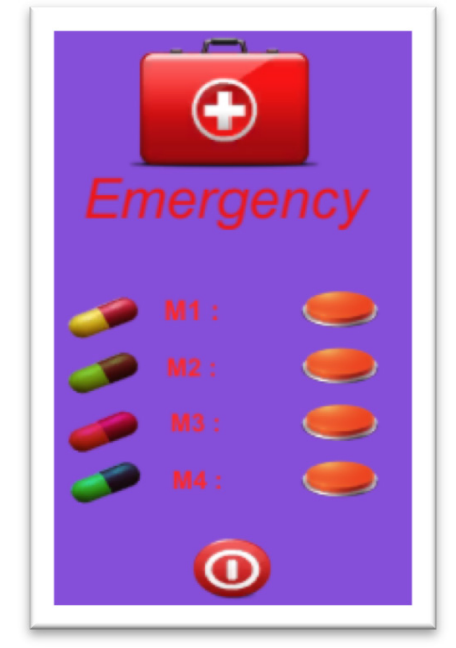

Fig. 17.Emergency layout

Admin page: In this page, the admin can set the password of the nurse and reset the admin's password, as shown in Fig. 16.

Emergency page: This is a page, as shown in Fig. 17, can be accessed by both the admin and the nurse. It has four buttons that can dispense the dosses immediately when the patient is in critical condition or missing one of their prescribed doses and needs medication immediately. The nurse can press any button and dispense the medicine.

\section{Conclusion}

A module and method were developed to increase medical adherence using both embedded technology and GSM services. A mobile application was developed to integrate embedded technology with GSM services. User interaction with the embedded system and tracking of dosage and reminder service is provided using a combination of embedded technology along with mobile application. Effective tracking of dosage is done using IR sensors and mobile application. The system provides an easy to use user interface for patients, nurses and doctors through mobile applications. Medical adherence can be improved through proper monitoring of medical dosage. The system reminds the patient to take right amount of medicine dosage at appropriate times through SMS.

\section{References}

[1] Abubakar Adam, Adamu Abubakar, Murni Mahmud (2019) "Sensor enhanced health information systems: issues and challenges," International Journal of 
[2] Interactive Mobile Technologies (iJIM), vol. 13, no. 1, pp 99-114. https://doi.org/10.3991/ijim.v13i01.7037

[3] El Mahjoubi A, Tomader Mazri, Nabil Hmina (2019) "NB-IoT and eMTC: Engineering results towards 5G/IoT mobile technologies," International Journal of

[4] Interactive Mobile Technologies (iJIM), vol. 13, no. 1, pp 115-126. https://doi.org/10.3991/ijim.v13i01.9728

[5] A Sinnemäki, J, Sinikka Sihvo, Jaana Isojärvi, Marja Blom, Marja Airaksinen and Antti Mäntylä (2013) "Automated dose dispensing service for primary healthcare patients: a systematic review," Systematic Reviews, vol. 2, no. 1, p. 1. https://doi.org/10.1186/20464053-2-1

[6] The Association of Finnish Pharmacies (2004) "Medicines in pre-packed doses." [Online]. Available: http://www.apteekkariliitto.fi/media/pdf/annual_report_2003.pdf. [Accessed 7 Jan 2019].

[7] Larsson A, Åkerlund M (2007) "ApoDos: the Swedish model of multi-dose," EJHP Pract., vol. 13, no. 51. Google Scholar

[8] Gombos, A. (2004) " Automated dose dispensing is a good system," Tidsskr Nor Laegeforen, vol. 124, no. 1144 .

[9] Apoteket, AB (2009) Annual report. [Online] Available: http://www.apoteket.se/privatpersoner/om/Documents/Finansiell\%20information/Apoteke t_2010_ENG_web\%20(2).pdf. [Accessed 7 Jan 2019].

[10] Finland Ministry of Social Affairs and Health "Safe pharmacotherapy for the elderly: Obligations for the municipalities," [Online] Available: http://www.stm.fi/tiedotteet/kuntainfot/kuntainfo/view/1236539. [Accessed 7 Jan 2019].

[11] Wooten J M (2012) "Pharmacotherapy Considerations in Elderly Adult," Southern Medical Journal, vol. 105, no. 8, pp. 437-445. [Online] Available: https://doi.org/10.1 097/SMJ.0b013e31825fed90

[12] The Association of Finnish Pharmacies (2003) "Medicines in pre-packed doses," Annual Review. [Online]: Available: http://www.apteekkariliitto.fi/media/pdf/annual_report_2003.pdf [Accessed 8 Jan 2019]

[13] The Palestinian Central Bureau of Statistics (PCBS) (2017) "Population in Palestine." [Online]. Available: http://www.pcbs.gov.ps. [Accessed 7 Jan 2019]

[14] Palestinian National Authority (2011) "Family Survey-Main Report," Palestinian Central Bureau of Statistics, pp. 1-10. http://www.pcbs.gov.ps.

[15] World Health Organisation (2016) "Occupied Palestinian territory: Country statistics summary." [Online]. Available: https://rho.emro.who.int/rhodata/node.country.countryPSE-stats?lang=en. [Accessed 7 Jan 2019].

[16] Husseini A, Abu-Rmeileh NME, Mikki N, Ramahi TM, Ghosh HA, Barghuthi N, Khalili M, Bjertness E, Holmboe-Ottesen G, Jervell J. (2009) "Cardiovascular diseases, diabetes mellitus, and cancer in the occupied Palestinian territory," Lancet, vol. 373, no. 9668, pp. 1041-1049. https://doi.org/10.1016/S0140-6736(09)60109-4

[17] Mansour, Muna and Abu Elhawa, Aisha (2013) "Aging in Palestine," This week in Palestine, pp. 14-17. [Online]. Available: https://www.thisweekinpalestine.com/i185/pdfs/article/aging_in_palestine.pdf

[18] Arduino Uno Rev3, Arduino UNO board. [Online]. Available: https://www.arduino.cc/en/Main/ArduinoBoardUno [Accessed 10 Jan 2018]

[19] Arduino Board, ESP8266 as an Arduino, [Online]. Available: https://www.arduinoboard.com/arduino/esp8266-arduino [Accessed 10 Jan 2018]

[20] Maxim Integrated (2015), DS1307-I2C Real-Time Clock datasheet. [Online]. Available: https://datasheets.maximintegrated.com/en/ds/DS1307.pdf [Accessed 10 Jan 2018] 
[21] Sensor Embedded, NEO-M590 GSM/GPRS industrial wireless module, [Online]. Available: http://sensorembedded.com/product_extra_files/Neo-M590\%20GSM,GPRS.pdf [Accessed 10 Jan 2018]

[22] QQ Trading, Motor driver L298N with Arduino board, [Online]. Available: http://qqtrading.com.my/stepper-motor-driver-module-L298N [Online]. Available: [Accessed 10 Jan 2018]

\section{$7 \quad$ Author}

Hatem Elaydi is member of the International Association of Online Engineering (IAOE). He is an associate professor of electrical engineering. He is the Associate Dean for Graduate Studies and Scientific Research at the Islamic University of Gaza. He is the Associate Editor-in-Chief of JERT. He is been an IEEE member since 1988.

Article submitted 2019-02-03. Resubmitted 2019-03-14. Final acceptance 2019-03-15. Final version published as submitted by the authors. 